\title{
Label-Free and Highly-Sensitive Detection of Ochratoxin A Using One-Pot Synthesized Reduced Graphene Oxide/Gold Nanoparticles-Based Impedimetric Aptasensor
}

\author{
Yasmin Alhamoud ${ }^{1}$, Yingying Li ${ }^{1}$, Haibo Zhou ${ }^{2}{ }^{\circledR}$, Ragwa Al-Wazer ${ }^{3}$, Yiying Gong ${ }^{1}$, Shuai Zhi ${ }^{1, *}$ \\ and Danting Yang ${ }^{1, *}$
}

check for

updates

Citation: Alhamoud, Y.; Li, Y.; Zhou, H.; Al-Wazer, R.; Gong, Y.; Zhi, S.; Yang, D. Label-Free and Highly-Sensitive Detection of Ochratoxin A Using One-Pot Synthesized Reduced Graphene Oxide/Gold Nanoparticles-Based Impedimetric Aptasensor. Biosensors 2021, 11, 87. https://doi.org/ 10.3390/bios11030087

Received: 3 February 2021

Accepted: 16 March 2021

Published: 19 March 2021

Publisher's Note: MDPI stays neutral with regard to jurisdictional claims in published maps and institutional affiliations.

Copyright: (c) 2021 by the authors. Licensee MDPI, Basel, Switzerland. This article is an open access article distributed under the terms and conditions of the Creative Commons Attribution (CC BY) license (https:// creativecommons.org/licenses/by/ $4.0 /)$
1 Zhejiang Key Laboratory of Pathophysiology, Department of Preventative Medicine, School of Medicine, Ningbo University, 818 Fenghua Road, Ningbo 315211, China; yasminalhamoud@zju.edu.cn (Y.A.); 176001260@nbu.edu.cn (Y.L.); 176001002@nbu.edu.cn (Y.G.)

2 Institute of Pharmaceutical Analysis and Guangdong Province Key Laboratory of Pharmacodynamic Constituents of Traditional Chinese Medicine \& New Drug Research, College of Pharmacy, Jinan University, Guangzhou 510632, China; haibo.zhou@jnu.edu.cn

3 Department of Pharmacy, Faculty of Applied Medical Sciences, Yemeni Jordanian University, 1833 Sana'a, Yemen; a_ragwa@outlook.com

* Correspondence: zhishuai@nbu.edu.cn (S.Z.); yangdanting@nbu.edu.cn (D.Y.)

\begin{abstract}
Ochratoxin A (OTA) primarily obtained by the genera aspergillus and penicillium, is one of the toxic substances for different organs and systems of the human body such as the kidney, liver, neurons and the immune system. Moreover, it is considered to cause tumors and fetal malformation even at a very low concentration. Fast and sensitive assay for detection of OTA at ultralow levels in foods and agricultural products has been an increasing demand. In this study, a new label-free electrochemical biosensor based on three-dimensional reduced graphene oxide/gold nanoparticles/aptamer for OTA detection was constructed. The 3D-rGO/Au NPs nanocomposites were firstly synthesized using a one-pot hydrothermal process under optimized experimental conditions. The 3D-rGO/Au NPs with considerable particular surface area and outstanding electrical conductivity was then coated on a glass carbon electrode to provide tremendous binding sites for -SH modified aptamer via the distinctive Au-S linkage. The presence of OTA was specifically captured by aptamer and resulted in electrochemical impedance spectroscopy (EIS) signal response accordingly. The constructed impedimetric aptasensor obtained a broad linear response from $1 \mathrm{pg} / \mathrm{mL}$ to $10 \mathrm{ng} / \mathrm{mL}$ with an LOD of $0.34 \mathrm{pg} / \mathrm{mL}$ toward OTA detection, highlighting the excellent sensitivity. Satisfactory reproducibility was also achieved with the relative standard deviation (RSD) of $1.393 \%$. Moreover, the proposed aptasensor obtained a good recovery of OTA detection in red wine samples within the range of 93.14 to $112.75 \%$ along with a low LOD of $0.023 \mathrm{ng} / \mathrm{mL}$, indicating its applicability for OTA detection in real samples along with economical, specific, susceptible, fast, easy, and transportable merits.
\end{abstract}

Keywords: Ochratoxin A; electrochemical biosensor; gold nanoparticles; three-dimensional reduced graphene oxide; aptamer

\section{Introduction}

Ochratoxin A is one of the most popular mycotoxins that is produced by two genera of fungi: Penicillium and Aspergillus, which can be found in many types of food consumed daily such as cereals, coffee, wine, spices, and dairy products [1-3]. Ochratoxin A (OTA) is found to cause severe harmful effects including genotoxic, neurotoxic, carcinogenic, and nephrotoxic effects on both animals and humans [4]. Accordingly, there is an urgent necessity to find accurate, selective, specific, and highly sensitive methods for detecting OTA even if it is found in very low concentrations. To this day, there are many accurate and highly efficient traditional methods such as chromatography (HPLC, LC/MS) [5,6] and enzyme-linked immunosorbent assay (ELISA) [7] that have been widely used to 
detect OTA in food, but the limitations of these methods are that they require highly skilled personnel, advanced equipment and time-consuming preparation and detection. Recently, electrochemical biosensors have attracted great attention by researchers as a reliable and fast point-of-care application in various fields. Furthermore, the occurrence of aptamers as the remarkable recognition probes with high specificity and affinity, fast response, simple operation, low cost, and miniaturization, have been used as convenient, sensitive, and robust methods for OTA monitoring with the necessary help of advanced nanomaterials in foodstuffs [8,9]. The electrochemical aptasensors are classified into labelfree and label-based types relying on whether the detection signals are from redox-labelled aptamers [10] or the redox probe in the electrolyte [11]. The redox-labelled aptamer EC sensor exhibits high sensitivity; however, the complex construction and high cost limited its development [12]. Thus, the label-free EC aptasensor based on a redox probe such as $\left[\mathrm{Fe}(\mathrm{CN})_{6}\right]^{3-/ 4-}$ or MB in the electrolyte was developed to overcome the above problem [13]. However, low stability of aptamer immobilized on the electrode and low sensitivity of detection signals are still critical problems in label-free based aptasensor construction.

Graphene has been widely used in the fabrication of electrochemical biosensors due to its outstanding physical properties and electrical conductivity [14]. However, the surface development is still intrinsically restricted by the two-dimensional (2D) nature which suffers from the poor processability and the agglomeration or re-stacking of graphene nanosheets that diminishes its intrinsic properties (electron transport and restricts application) [15] In order to avoid the re-stacking of individual sheets, some endeavors have been made recently to develop three-dimensional (3D) structure graphene $[16,17]$. These unique interconnected network structures of 3D graphene improve the properties and features of 2D graphene, giving it high electrical conductivity, large surface area, high porosity, and distinct mechanical merits $[18,19]$. Reduced graphene oxide (rGO), allowing more immobilized nanoparticles to be deposited on its large surface area with high stability, has been preferred in the electrochemical aptasensors construction over graphene [20,21]. Moreover, gold nanoparticles (AuNPs) can be embedded in rGO to construct aptasensor with a highly charge transfer rate and considerable active surface area [22] along with promisingly biocompatibility for anchoring thiolated/sulfhydryl DNA [23,24]. For instance, the AuNPs dotted rGO nanocomposites-based electrochemical aptasensors could achieve a high ultrasensitive detection of miRNA with a LOD of $1.73 \mathrm{pM}$ [25]. In this regard, the 3D-rGO/Au NPs nanocomposites will be an excellent immobilization substrate for aptamer and high charge transporter for the development of ultrasensitive label-free based biosensor.

There are two different ways to fabricate rGO-AuNPs nanocomposites, including ex situ hybridization and in situ decoration [26]. For instance, the rGO-AuNPs nanocomposites used for mycotoxin [27] or OTA detection [8,28] were synthesized via ex situ hybridization process, which means the AuNPs nanoparticles were prepared in advance and then adsorbed onto the surface of RGO through either covalent or noncovalent binding. In comparison with the ex situ hybridization process, the preparation of the in-situ synthesis approach is much easier [29], which could facilitate the fabrication process of the electrochemical electrode. Currently, there is no report of label-free based electrochemical aptasensor based on one-pot, in-situ decorated 3D-rGO/AuNPs nanocomposites for highly efficient and ultrasenstive detection of OTA in wine. In this work, we designed a novel impedimetric aptasensor using one-pot, in-situ synthesized 3D-rGO/Au NPs nanocomposites toward label-free and sensitive OTA determination. Parameters for 3D-rGO/Au NPs nanocomposites synthesis were optimized to provide high sensitivity and stability. The aptamer-3D-rGO/Au NPs/GCE system was formed via the Au-S covalent bond between the specific OTA aptamer and 3D-rGO-Au NPs/GCE substrate. When OTA is present, it will bind with aptamer, forming an OTA-aptamer curled complex on the surface of the modified electrode that causes the corresponding EC signal responses of $\left[\mathrm{Fe}(\mathrm{CN})_{6}\right]^{3-/ 4-}$ redox probe. The proposed OTA aptasensor showed ultrasensitive detection limit, good reproducibility, high stability, and good recovery in real samples. 


\section{Materials and Methods}

\subsection{Materials and Chemicals}

Methanol and potassium chloride $(\mathrm{KCl})$ were purchased from the Aladdin company (Shanghai, China), potassium ferrocyanide $\left(\mathrm{K}_{4}\left[\mathrm{Fe}(\mathrm{CN})_{6}\right]\right)$, potassium ferricyanide $\left(\mathrm{K}_{3}\left[\mathrm{Fe}(\mathrm{CN})_{6}\right]\right)$, disodium hydrogen phosphate dodecahydrate $\left(\mathrm{Na}_{2} \mathrm{HPO}_{4}-12 \mathrm{H}_{2} \mathrm{O}\right)$, disodium hydrogen phosphate dehydrate $\left(\mathrm{NaH}_{2} \mathrm{PO}_{4}-2 \mathrm{H}_{2} \mathrm{O}\right)$, sodium hydroxide $(\mathrm{NaOH})$, and glucose $\left(\mathrm{C}_{6} \mathrm{H}_{12} \mathrm{O}_{6}\right)$, were purchased from Sinopharm Chemical Reagent Co., Ltd. (Shanghai, China). Chloroauric acid $\left(\mathrm{HAuCl}_{4} \cdot 4 \mathrm{H}_{2} \mathrm{O}\right.$ ), graphene oxide nanosheets (GO, XF002-1, $500 \mathrm{~nm}-5 \mu \mathrm{m}$; $\sim 99 \%$, Hummers) were purchased from Nanjing XFNANO Materials Tech. Co., Ltd (Nanjing, China). Tris(2-carboxyethyl) phosphine (TCEP) was obtained from Sigma-Aldric. Ochratoxin A (OTA), ochratoxin B (OTB), deoxynivalenol (DON), and zearalenone (ZEA) were purchased from Sigma-Aldrich (Shanghai, China). Tris(hydroxymethyl)aminomethane hydrochloride (tris- $\mathrm{HCl}$ ) and ethylenediaminetetraacetic acid disodiumsalt (EDTA) were purchased from Sinopharm Chemical Reagent Co., Ltd. (Shanghai, China). Bovine serum albumin (BSA) was purchased from Sigma-Aldrich. OTA binding-aptamer with a sequence (5'-GAT CGG GTGTGG GTG GCG TAA AGG GAG CAT CGG ACA- $\left(\mathrm{CH}_{2}\right)_{6}-\mathrm{SH}-3^{\prime}$ ) was purchased from Sangon Biotech. (Shanghai, China). All reagents and chemicals were of the highest analytical grade. Millipore-Q water $\left(18.2 \mathrm{M} \Omega \mathrm{cm}^{-1}\right)$ was used to prepare all aqueous solutions.

\subsection{Synthesis of $3 D-r G O / A u$ NPs Nanocomposites}

3D-rGO/Au NPs nanocomposites were fabricated through a one-pot hydrothermal reduction process using $\mathrm{GO}$ nanosheets, $\mathrm{HAuCl}_{4}$, and glucose. Key parameters for 3D$\mathrm{rGO} / \mathrm{AuNPs}$ such as amounts of glucose and $\mathrm{HAuCl}_{4} \cdot 4 \mathrm{H}_{2} \mathrm{O}$, were optimized to obtain well-dispersed and long-stabilized 3D-rGO/AuNPs. In brief, a $2 \mathrm{mg} / \mathrm{mL}$ suspension of GO was prepared by the sonication of GO nanosheets $(20 \mathrm{mg})$ in water $(10 \mathrm{~mL})$ for $2 \mathrm{~h}$. Then, the above dispersion was mixed with $10 \mathrm{~mL}$ glucose $(2,50$, and $150 \mathrm{mg} / \mathrm{mL})$ solution under sonication. The volume $(200,400,1000$, and $1600 \mu \mathrm{L})$ of $\mathrm{HAuCl}_{4} \cdot 4 \mathrm{H}_{2} \mathrm{O}(2 \%, w / w)$ was introduced into the mixture and sonicated evenly for $1 \mathrm{~h}$. Afterwards, the obtained homogenous solution was poured into a Teflon-lined autoclave and reacted at $180{ }^{\circ} \mathrm{C}$ for a full $12 \mathrm{~h}$. After the autoclave was cooled to the room temperature, the aerogel was washed three times using distilled water and blotted with filter paper to remove surfaceadsorbed water. At last, the obtained 3D-rGO/Au NPs hydrogel was freeze-dried $\left(-50^{\circ} \mathrm{C}\right)$ for $48 \mathrm{~h}$ to get the dry 3D-rGO/Au NPs nanocomposites. For comparison of rGO and $\mathrm{rGO} / \mathrm{AuNPs}$, $\mathrm{rGO}$ was synthesized with the same protocol of $\mathrm{rGO} / \mathrm{AuNPs}$ without the addition of $\mathrm{HAuCl}_{4} \cdot 4 \mathrm{H}_{2} \mathrm{O}$. In addition, the role of glucose and high temperature in the thermal reduction was investigated.

\subsection{Preparation of the Aptasensor Surface}

Firstly, the glassy carbon electrode (GCE) was polished with alumina powder (diameters 1.0 and $0.05 \mu \mathrm{m}$ ) until a mirror-like surface was obtained and then was sonicated in water and absolute ethanol consequently. After the cleaned electrode was dried at room temperature, $8.0 \mu \mathrm{L}$ of 3D-rGO-AuNPs nanocomposite suspension was drop-casted onto the surface of GCE and left to dry for about $2 \mathrm{~h}$ at room temperature. Subsequently, the aptamer was activated by $100 \mathrm{mM}$ TCEP and incubated for $1 \mathrm{~h}$ at $37^{\circ} \mathrm{C}$. Then $10 \mu \mathrm{L}$ of activated aptamer was dropped on the surface of the modified electrode. After incubated for $2 \mathrm{~h}$ at room temperature, the unbound aptamers were excluded from the modified electrode surface by careful washing with phosphate buffered saline (PBS) solution three times. After drying, the obtained aptasensor was stored at $4{ }^{\circ} \mathrm{C}$ for the following experiments.

\subsection{Electrochemical Measurement}

In order to monitor the changes occurring effectively and comprehensively on the modified electrode surface both electrochemical impedance spectroscopy (EIS) and cyclic voltammetry $(\mathrm{CV})$ were used. EIS was recorded in $0.1 \mathrm{M}$ PBS buffer with $\mathrm{pH} 7.4$ in the 
presence of $1 \mathrm{mM}\left[\mathrm{Fe}(\mathrm{CN})_{6}\right]^{3-/ 4-}$ and $0.1 \mathrm{M} \mathrm{KCl}$ at a bias potential of $+0.20 \mathrm{~V}$, an amplitude of $5 \mathrm{mV}$ and a frequency range from 0.1 to $10^{6} \mathrm{~Hz}$. CV measurements of various fabricated electrodes were performed with potential from -0.6 to $+0.6 \mathrm{~V}$ and scan rate of $100 \mathrm{mV} / \mathrm{s}$. For OTA detection, the prepared aptasensor was dipped in a 0.1 M PBS buffer (pH 7.4) containing OTA for $1 \mathrm{~h}$. Then OTA-captured electrodes were rinsed with PBS buffer three times. After drying at room temperature, the EIS measurements were performed to detect OTA.

\subsection{Instrumentation}

All the electrochemical measurements were conducted using a model CHI 660D electrochemical workstation (Shanghai Chenhua Instruments Co. Ltd., Shanghai, China). A 3-mm-diameter GCE was used as the working electrode, a platinum electrode as the auxiliary electrode, and an $\mathrm{Ag} / \mathrm{AgCl}$ saturated with $\mathrm{KCl}$ as the reference electrode are the system elements that composed the three-electrode system for electrochemical experiments. All the potentials were reported with respect to the reference electrode. The structure and morphology of synthesized nanocomposites was checked by scanning electron microscopy (SEM) (S-3000N, Hitachi, Tokyo, Japan) and transmission electron microscopy (TEM) (H-7650, Hitachi, Tokyo, Japan). Energy-dispersive X-ray spectroscopy (EDS) elementary analysis was performed with the VEGA-3-SBH (Tescan, Brno, Czech). X-ray diffraction (XRD) patterns were carried out with the D8 Advance X-ray powder diffractometer (Bruker, Berlin, Germany). Raman spectroscopy was recorded by a Raman microscope with a 532-laser source from 800 to $3600 \mathrm{~cm}^{-1}$, with an exposure time of $1 \mathrm{~s}$ and an accumulation number of 10. UV-vis spectra were obained with a D8 Spectrometer (Feile Instruments, Nanjing, China). Drying of 3D-rGO/AuNPs nanocomposites under vacuum was done with a Freeze dryer (Ningbo Shuangjia Instruments, Ningbo, China). The $\mathrm{pH}$ measurements were done with a Metrohm model $691 \mathrm{pH} / \mathrm{mV}$ meter (Metrohm, Herisau, Switzerland).

\section{Results}

\subsection{Principles of 3D-rGO/Au NPs Nanocomposites Based Label-Free Aptasensor for OTA}

As described in Figure 1, a label-free and highly sensitive impedimetric aptasensor for detection of OTA based on 3D-rGO/AuNPs nanocomposites was designed. $1 \mathrm{mM}$ $\left[\mathrm{Fe}(\mathrm{CN})_{6}\right]^{3-/ 4-}$ with $0.1 \mathrm{M} \mathrm{KCl}$ in the PBS solution was used as the redox probe to record the EIS response of every step. Firstly, the surface of GCE was modified by drop-coating nanocomposites of 3D-rGO-AuNPs. The value of the charge transfer resistance decreased compared with the bare GCE due to the great conducting ability and the increased electron transfer rate of rGO and AuNPs. The resultant film was then bound with aptamer via Au-S covalent bonds and formed the GCE-3D-rGO-AuNPsaptamer system. Here, the electron transfer is hampered by its isolating effect, leading to the increase of the charge-transfer resistance of the electrode. Then the modified electrode was incubated with 1.0\% BSA to block any possible non-specific binding as commonly used in biosensing $[30,31]$. When OTA-aptamer was specifically combined on the aptasensing surface, this obtained the curled complex of OTA-aptamer which partly blocked the electron transfer path and then the EIS signal responses changed with the according OTA concentration.

Glucose with abundant oxidative groups and nontoxic merit, was shown to be an excellent reducing agent to reduce GO into rGO in the hydrothermal process [32,33] and to reduce $\mathrm{Au}^{3+}$ to $\mathrm{Au}$ [29] as well. As shown in Figure S1, the sample "b" was synthesized without high temperature in the presence of glucose (detailed parameters can be seen in Table S1) and exhibited the yellow-brown color of GO and a much lower CV current peak than that of sample "c" (Figure S2D); this indicated that sample b was not reduced compared to the dark color of our proposed sample " $\mathrm{C}$ " at high temperature. Furthermore, with the fabrication of sample " $b$ " on the GCE, the Rct was larger than the bare GCE (Figure S2A), indicating the insulating property of sample " $\mathrm{b}$ ". It can be concluded that glucose can only perform its reduction role under high temperature to synthesize rGO/AuNPs. In 
addition, under high temperature, GO could be reduced to rGO despite the absence of glucose and enabled the analysis of OTA through layer-by-layer fabrication as seen in Figure S2C. However, the maximum current peak of the CV curves of sample " $c$ ", synthesized with the presence of glucose, is 1.3 fold higher than that of sample " $\mathrm{d}$ " (Figure S2D). Moreover, the absence of glucose could cause the rGO sentiment in $5 \mathrm{~min}$ as shown in sample " $\mathrm{d}$ ", compared with our proposed stable sample " $\mathrm{c}$ " in one month. In comparison of $\Delta \mathrm{R}_{\mathrm{CT}}$ between BSA and OTA fabrication on GCE (Figure S2B,C), it was found that the $\Delta \mathrm{R}_{\mathrm{CT}}$ (OTA-BSA) of sample " $c$ " (52 $\pm 5 \Omega)$ is larger than that of sample " $d$ " (34 $\pm 10 \Omega)$ with smaller relative standard deviation (RSD), which indicates the glucose performs as the stabilizer in the synthesis of $\mathrm{rGO} / \mathrm{AuNPs}$ in addition to reducing agent [29].

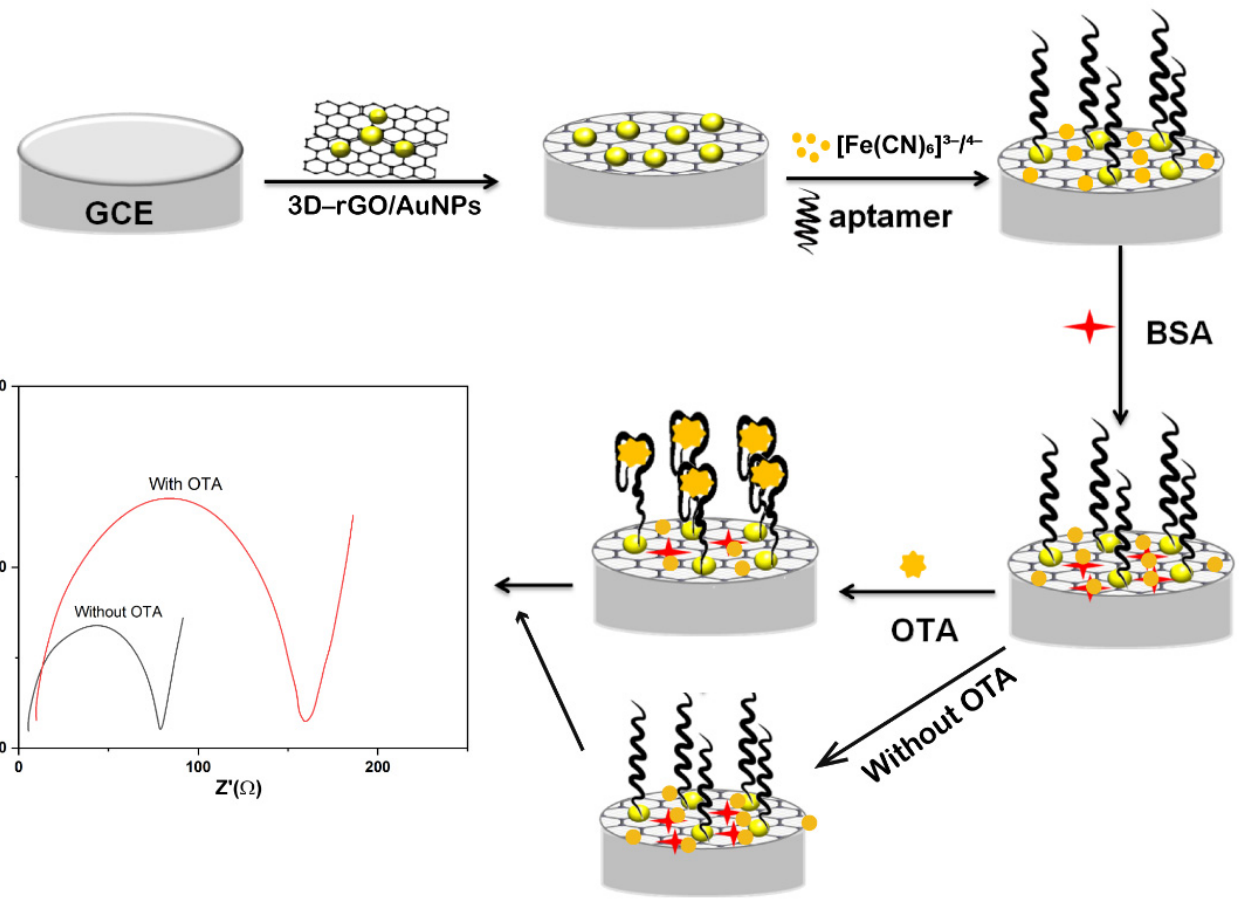

Figure 1. Schematic illustration of GCE/3D-rGO/AuNPs based aptasensor for Ochratoxin A (OTA).

\subsection{Optimization and Characterization of 3D-rGO/AuNPs Nanocomposites}

As the critical role of gold amounts and glucose in the system, the amounts of $\mathrm{HAuCl}_{4}$ $(200,400,1000$, and $1600 \mu \mathrm{L})$ (Table S2) and glucose (20, 500, and $1500 \mathrm{mg}$ ) (Table S3) were firstly optimized to obtain the largest charge-transfer resistance change and the fastest ion diffusion ability, respectively. EIS was used to investigate the characteristics of charge and ion transfer in the 3D-rGO/AuNPs at different synthesized conditions in a frequency range of $10^{6} \mathrm{~Hz}$ to $0.1 \mathrm{~Hz}$. The diameter of the semicircle in the high-frequency range gives an approximate value of the charge-transfer resistance (Rct) at the electrode/electrolyte interface and the straight slope line in the low-frequency region is ascribed to the diffusive resistance of the electrolyte in the electrode pores and proton diffusion in the host materials. It can be seen in Figure 2A, sample " $b$ " showed a more vertical straight line than samples "a, c, and d", indicating the faster ion diffusion ability of the porous rGO materials. We can also see from the Figure 2B, the sample "a" exhibited the smallest semicircle among sample "a, b, c and bare GCE", suggesting the loading of sample "a" onto the GCE surface resulted in the highest decrease in charge-transfer resistance to bare GCE. Thus, the sample exhibiting the most vertical straight line along with the smallest semicircle was chosen as the optimized nanomaterial, which means the synthesized condition was set as $400 \mu \mathrm{L}$ of $\mathrm{HAuCl}_{4}, 20 \mathrm{mg}$ glucose, and $20 \mathrm{mg}$ GO in $20 \mathrm{~mL}$ aqueous solution. 

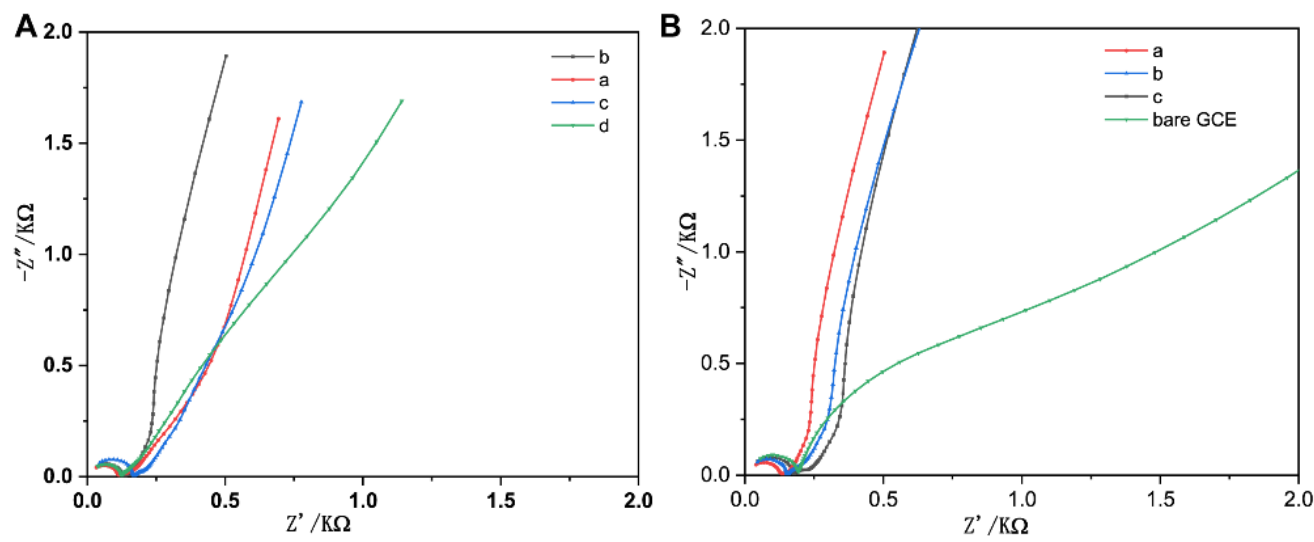

Figure 2. Electrochemical impedance spectroscopy (EIS) measurements of (A) different volumes of $\mathrm{HAuCl}_{4} \cdot 4 \mathrm{H}_{2} \mathrm{O}(\mathrm{a}, 200 \mu \mathrm{L} ; \mathrm{b}, 400 \mu \mathrm{L} ; \mathrm{c}, 1000 \mu \mathrm{L} ; \mathrm{d}, 1500 \mu \mathrm{L})$ and (B) different amounts of glucose (a, $20 \mathrm{mg}$; b, $500 \mathrm{mg}$; c, $1000 \mathrm{mg}$ ) for 3D-rGO/AuNPs nanocomposites and bare glassy carbon electrode (GCE).

\subsubsection{Morphology and Structure Characterization of 3D-rGO/AuNPs Nanocomposites}

SEM and TEM have been used to characterize the morphology and structure of synthesized nanomaterials. The SEM and TEM images of 3D-rGO/AuNPs nanomaterials are illustrated in Figure 3A,C,D, respectively. As shown in Figure 3A, the nanocomposite has high porosity and a sponge-like structure with AuNPs clearly embedded in it. Compared with the TEM image of rGO in Figure 3B, the distribution of AuNPs (black dots, size of $\sim 140 \mathrm{~nm}$ shown in Figure 3D) in the porous structure of rGO is clearly seen in Figure 3C, which is important and necessary for chemisorption of aptamer during the immobilization step via Au-S linkage. According to the EDS elemental analysis (Figure 3E), the presence of $\mathrm{Au}, \mathrm{C}, \mathrm{O}$ in our nanomaterial was evidenced along with the percentage of Au NPs content of $1.74 \%$. UV - vis absorption spectrometry was also used to identify the presence of rGO and AuNPs. As was shown in Figure 3F the pure GO exhibited a typical absorption peak at $230 \mathrm{~nm}$. After the hydrothermal process, the $230 \mathrm{~nm}$ peak was shifted to $265 \mathrm{~nm}$, indicating the change from GO to rGO, which could be seen in both $\mathrm{rGO}$ and $\mathrm{rGO} / \mathrm{AuNPs}$. The second peak at $538 \mathrm{~nm}$ was assigned to Au NPs. This result accords to the previous report [29,34], illustrating the successful formation of rGO/AuNPs.

For further investigation, XRD was applied to study the purity and crystallinity of 3D-rGO-AuNPs nanomaterials. Figure 4A demonstrates the wide angle XRD profiles of 3D$\mathrm{rGO} / \mathrm{AuNPs}$. As we can see, the pattern of the sample contains broad reflections at $2 \theta=\sim 20$ and $30^{\circ}$, which means that non-defect and high crystalline graphene was synthesized [21], indicating the formation of the $3 \mathrm{D}-\mathrm{rGO}$ structure during the hydrothermal process. The five well-resolved diffraction peaks at $2 \theta$ value of $\sim 38,44,64,77$, and $81^{\circ}$ can be indexed as monoclinic Au 111, 200, 220, 311, and 222 reflections, exhibiting the formation and presence of AuNPs. The Raman technique is one of the most efficient and nondestructive methods that enables investigating the quality and structure of carbon compounds. The Raman spectrum of 3D-rGO-AuNPs nanocomposite is shown in Figure 4B, exhibiting two prominent peaks at 1349 and $1600 \mathrm{~cm}^{-1}$, which correspond to the $\mathrm{D}$ and $\mathrm{G}$ bands of $\mathrm{rGO}$, respectively. The $\mathrm{D}$ band indicates the presence of defects caused by the $\mathrm{sp}^{3}$ hybridized carbon atoms, while the $\mathrm{G}$ band is a typical peak exhibited by the pristine $\mathrm{sp}^{2}$ lattice carbon atoms in the graphene sheet. The broadened 2D peak at $2700 \mathrm{~cm}^{-1}$ indicates that $\mathrm{GO}$ was reduced and composed of multi-layers of stacked graphene nanosheet [35-38]. In addition, the anodic peak current of bare GCE was reduced from $76.2 \mu \mathrm{A}$ to about $0.9 \mu \mathrm{A}$, which was due to the insulating properties of non-reduced GO layer on GCE resulting in the repulsion of $\left[\mathrm{Fe}(\mathrm{CN})_{6}\right]^{3-/ 4-}$ on the GCE surface ${ }^{-}$. However, when GCE was modified with $\mathrm{rGO},\left[\mathrm{Fe}(\mathrm{CN})_{6}\right]^{3-/ 4-}$ could diffuse through the porous structure of reduced nanomaterial and improve the redox activity. The electro-conductivity of GCE was further improved with the 3D-rGO/AuNPs nanomaterial as seen by the highest peak 
current of $128 \mu \mathrm{A}$ in Figure 4C, attributing to the synergic conductive performance of rGO and AuNPs which is in accordance with results in the published articles [29,39].
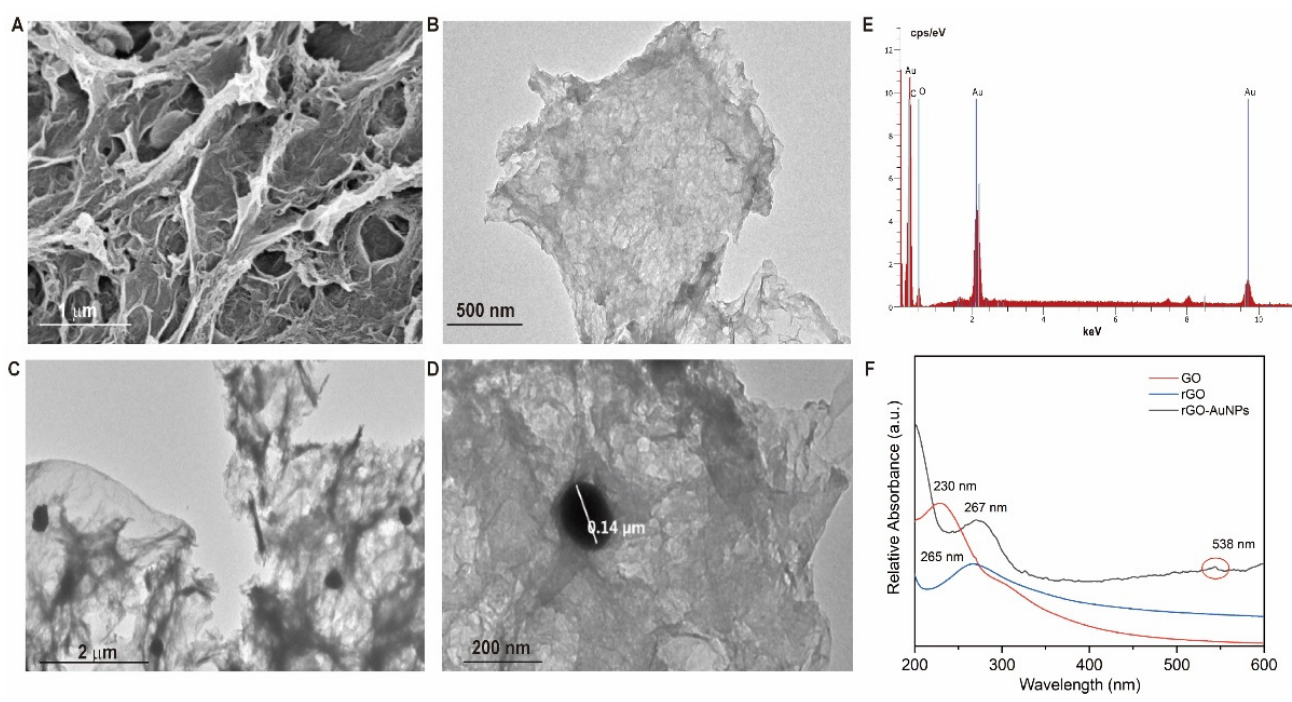

Figure 3. (A,B) Scanning electron microscopy (SEM) images of the prepared of 3D-rGO/AuNPs at different magnifications; (C,D) transmission electron microscopy (TEM) images of reduced graphene oxide (RGO) and rGO-AuNPs; (E) energy-dispersive X-ray spectroscopy (EDS) of 3DrGO/AuNPs; and (F) UV-vis spectra of graphene oxide (GO), reduced graphene oxide (rGO) and rGO-AuNPs nanocomposites.
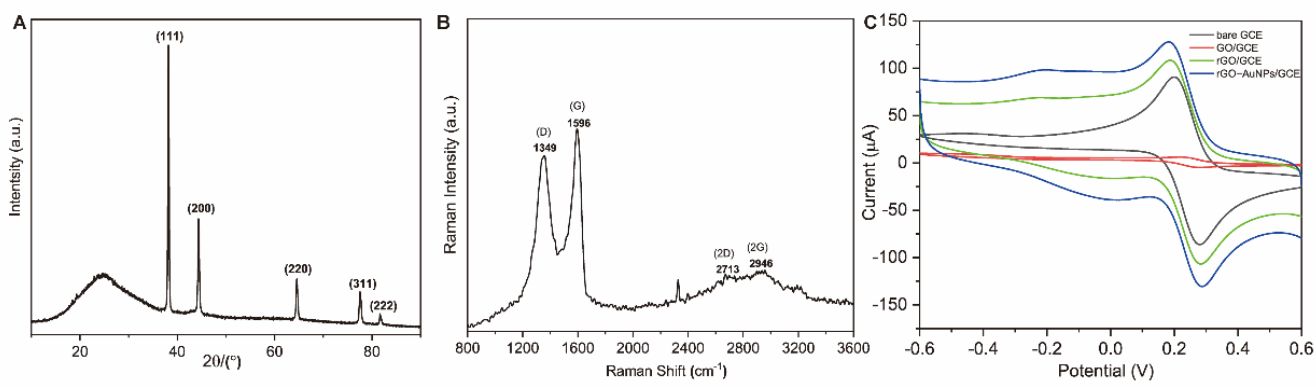

Figure 4. (A) X-ray diffraction (XRD) patterns of 3D-rGO/AuNPs nanocomposites; (B) Raman spectrum of 3D-rGO/AuNPs nanocomposites; (C) cyclic voltammetry (CV) curves of bare GCE, GO/GCE, 3D-rGO/GCE, and 3D-rGO-AuNPs/GCE.

\subsubsection{Electrochemical Characterization of the Aptasensor}

\section{(1) EIS measurements}

EIS is one of the effective methods applied to monitor the changes occurring on the modified electrode surface during various modification steps and thus verifying the success of the modification or immobilization processes. Nyquist plots were chosen to represent

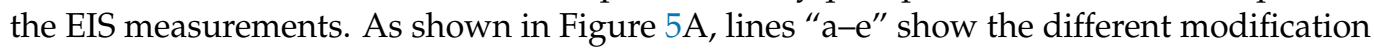
levels which was performed on the electrode surface. As evident, the semicircle diameter of line "a" is larger than that of line " $\mathrm{b}$ ", suggesting that the loading of 3D-rGO-AuNPs onto GCE results in a decrease of charge-transfer resistance. $R_{C T}$ value dramatically increased (shown as line " $\mathrm{c}$ ") after the aptamer was captured on the 3D-rGO-AuNPs-modified electrode surface, which could be interpreted as a result of the repulsion between the negatively charged $\left[\mathrm{Fe}(\mathrm{CN})_{6}\right]^{3-/ 4-}$ present in the standard solution and the negatively charged phosphate present in the aptamer structure. The $\mathrm{R}_{\mathrm{CT}}$ value of Line " $\mathrm{d}$ " slightly increased from $69 \pm 8$ to $72 \pm 5$ after incubation with BSA for non-specific blocking, which indicating BSA will not affect the sensitivity of the system. Finally, after the OTA presented, 
the $R_{C T}$ value increased significantly (Figure $5 \mathrm{~A}(\mathrm{e})$ ), which refers to the fact that OTA was captured by its aptamer, thus blocking the reaction sites on the sensing surface.
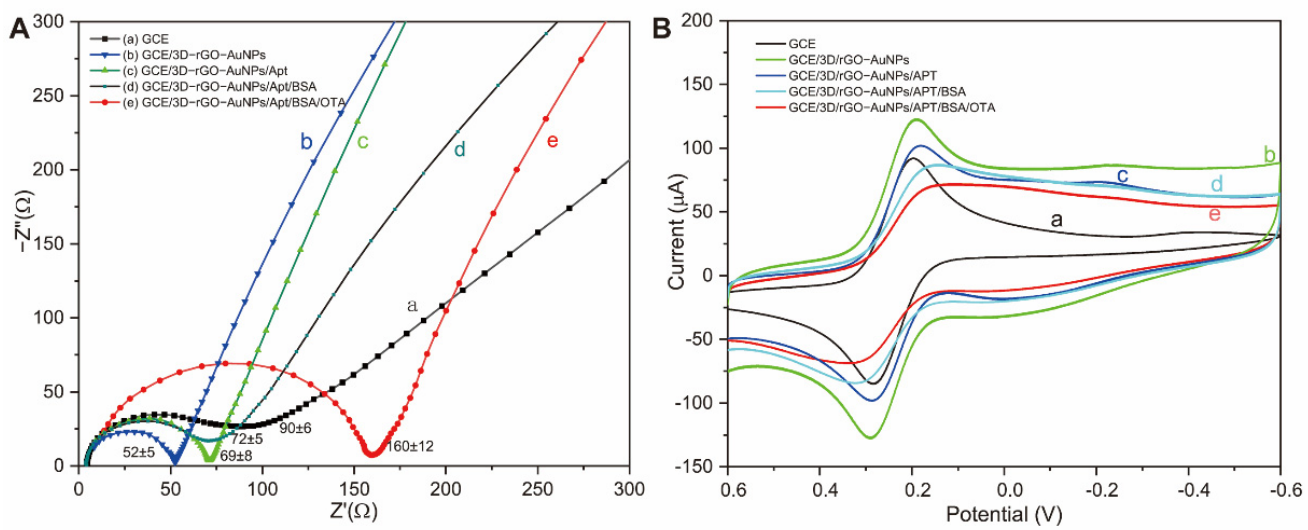

Figure 5. (A) Nyquist plots and (B) CV curves of (a) the bare GCE, (b) GCE/3D-rGO-AuNPs, (c) GCE/3D-rGO-AuNPs/aptamer, (d) GCE/3D-rGO-AuNPs/aptamer/BSA, and (e) GCE/3D-rGOAuNPs/aptamer/BSA/OTA in a solution of phosphate buffered saline (PBS) pH 7.4 containing $1 \mathrm{mmol} \mathrm{L}^{-1}\left[\mathrm{Fe}(\mathrm{CN})_{6}\right]^{3-/ 4-}$ and $0.1 \mathrm{~mol} \mathrm{~L}^{-1} \mathrm{KCl}$.

\section{(2) Cyclic voltammetry (CV)}

$\mathrm{CV}$ measurements, another efficient method for monitoring the interface properties of the sensor surface, were also taken. Figure $5 \mathrm{~B}$ shows the $\mathrm{CV}$ curves of the modified electrodes at different stages. After the 3D-rGO/AuNPs suspension was deposited on the bare electrode surface, the CV peak current obviously increased (Figure 5B), which indicates the coverage of electrode surface with a layer of 3D-rGO/AuNPs nanocomposite. The modified film facilitates the electrons transfer of the $\left[\mathrm{Fe}(\mathrm{CN})_{6}\right]^{3-/ 4-}$ pair and enables a larger amount of loaded aptamer by providing a greater specific surface area. When aptamer was incubated with the above modified electrode, the peak current patently decreased due to the role of the aptamer acting as an isolating barrier to electron transfer. (Figure 5B (c)), illustrates the successful immobilization of aptamer on the electrode. After incubation of BSA, a small decrease is shown, indicating the successful fabrication of blocking BSA (Figure 5B (d)). An additional significant decrease in the peak current value occurred due to increased electron transfer resistance after the incubation with OTA, (Figure 5B (e)), suggesting that the proposed OTA aptasensor was successfully achieved.

\subsection{Optimization of Aptasensing Parameters}

To obtain the best analytical performance of the proposed strategy, the most important testing variables such as 3D-rGO/AuNPs concentration, aptamer concentration, aptamer incubation time on the electrode, and aptamer-OTA incubation time were optimized, respectively. EIS is very sensitive to changes in interfacial impedance occurring at the surface/electrolyte interface based upon biorecognition events [40]. Thus, the difference between the values of the charge-transfer resistance $\left(\Delta \mathrm{R}_{\mathrm{CT}}\right)$ of GCE after BSA and OTA incubation was used for the evaluation of aptasensor analytical performance. The concentration of OTA was set as $1 \mathrm{ng} \cdot \mathrm{mL}^{-1}$ for the optimization.

The concentration of 3D-rGO/AuNPs nanocomposites aqueous suspension fabricated on the GCE can influence processes. The effect of different concentrations $(0.1,0.3,0.5,1$, and $2 \mathrm{mg} / \mathrm{mL}$ ) of the nanocomposites on the response of the electrochemical aptasensor was investigated. It is clear in Figure S3A that the 3D-rGO/AuNPs suspension concentration of $0.5 \mathrm{mg} / \mathrm{mL}$ exhibits the highest value of the charge-transfer resistance $\left(\mathrm{R}_{\mathrm{CT}}\right)$. However, EIS from samples of 1 and $2 \mathrm{mg} / \mathrm{mL}$ were not obtained; the modified film of those fell into the solution before completing the examination. The high concentration of 3D-rGO/AuNPs nanocomposites may cause a thick and heavy film on the electrode surface, which leads to weak fixation for a long time needed by EIS operation. In contrast, the EIS response 
of $0.5 \mathrm{mg} / \mathrm{mL}$ 3D-rGO/AuNPs nanocomposites was satisfied based on the difference between the values of the charge-transfer resistance $\left(\mathrm{R}_{\mathrm{CT}}\right)$ of aptamer and OTA. Therefore, $0.5 \mathrm{mg} / \mathrm{mL}$ was selected as the optimum concentration of 3D-rGO/AuNPs suspension for the following experiments.

The concentration of aptamer is one of the analytical variables that may affect the aptasensor response significantly. Therefore, we compared different concentrations of aptamer $(0.1,0.5,0.8,1$, and $2 \mu \mathrm{M})$ during the fabrication process. Figure S3B shows the effects of different-concentrations of aptamer on the electrochemical signal of the aptasensor by using $1 \mathrm{ng} / \mathrm{mL}$ OTA as the target. The EIS response was gradually increased from 0.1 to $1 \mu \mathrm{M}$ owing to the filling out of whole active sites of the 3D-rGO/AuNPs modified electrode. The saturation level of the modified electrode surface was inferred by adopting the aptamer concentration of $1 \mu \mathrm{M}$, which is selected as the optimized parameter for the following experiments.

The time of incubating aptamer with the electrode surface was optimized as well. As seen in Figure $\mathrm{S} 3 \mathrm{C}$, the $\mathrm{R}_{\mathrm{CT}}$ value magnified with increasing time and achieved a maximum value at $180 \mathrm{~min}$. Consequently, $180 \mathrm{~min}$ was adopted as the optimum aptamer incubation time. The incubation time of the OTA with its aptamer is also one of the most important parameters that impacts the analytical condition of the presented aptasensor. Finally, different OTA-aptamer incubation time from 10 to 90 min were examined, respectively. As shown in the Fig S3D, the $\mathrm{R}_{\mathrm{CT}}$ value increased notably with the increasing incubation time of OTA and reached the highest value (saturation level) at $60 \mathrm{~min}$. The $\mathrm{R}_{\mathrm{CT}}$ value decreased when the incubation time of OTA further increased. In order to get an aptasensor with fast response to OTA, $60 \mathrm{~min}$ was confirmed as the best incubation time.

Thus, $0.5 \mathrm{mg} / \mathrm{mL}$ of 3D-rGO/AuNPs suspension, $1 \mu \mathrm{M}$ of aptamer, $180 \mathrm{~min}$ of incubating aptamer with the electrode, and $60 \mathrm{~min}$ of incubating OTA with its aptamer were chosen as the optimal parameters.

\subsection{Analytical Performance of Electrochemical Aptasensors for OTA}

The analytical performance of the proposed electrochemical aptasensor for OTA detection of different concentrations $(0,0.001,0.01,0.05,0.1,1$, and $10 \mathrm{ng} / \mathrm{mL})$ under the optimum conditions were explored through EIS measurements. It is shown in Figure 6A, with the increasing concentration of OTA, the EIS responses were enhanced accordingly. By analyzing the results, we found that there is a good linear relationship between the value of $\Delta \mathrm{R}_{\mathrm{CT}}$ vs. the value of the logarithm of OTA concentration (log $\left.\left(\mathrm{c} / \mathrm{ng} \cdot \mathrm{mL}^{-1}\right)\right)$, which is shown in Figure 6B. The linear correlation equation can be indicated as $\Delta \mathrm{R}_{\mathrm{CT}}=88.73+24.90$ $\log \left(\mathrm{c} / \mathrm{ng} \cdot \mathrm{mL}^{-1}\right)\left(\mathrm{R}^{2}=0.997\right)$. The detection limit of OTA $(\mathrm{S} / \mathrm{N}=3)$ was $0.34 \mathrm{pg} / \mathrm{mL}$. The present aptasensor in this study had a lower LOD, which indicated that this technique was preferable in the detection of OTA. (Comparison of the developed impedimetric aptasensor with those from previous reports are summarized in Table 1)
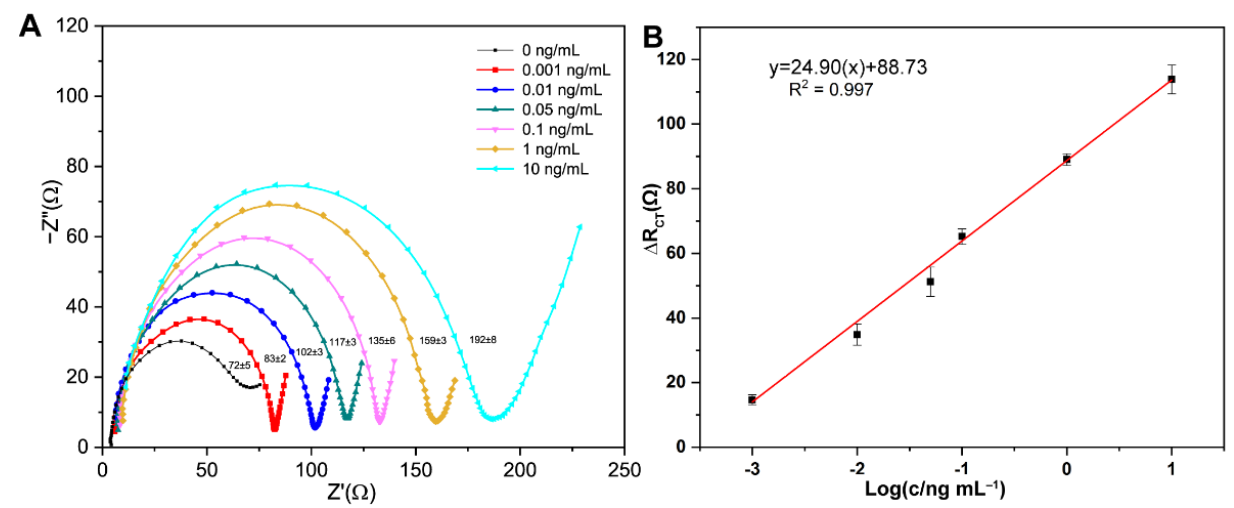

Figure 6. (A) Nyquist plots of aptasensor with different concentrations of OTA (0-10 ng/mL) and (B) The calibration curve of aptamer corresponding to the detection of OTA based on the change in electron-transfer resistance $\left(\mathrm{R}_{\mathrm{CT}}\right)$, which is presented as $\Delta \mathrm{R}_{\mathrm{CT}}$. 
Table 1. Comparison of the developed impedimetric aptasensors for OTA detection.

\begin{tabular}{|c|c|c|c|}
\hline Transducer & Linear Range (ng $\left.\cdot \mathrm{mL}^{-1}\right)$ & LOD (ng. mL $\left.{ }^{-1}\right)$ & Reference \\
\hline Thionine and $\mathrm{IrO}_{2}$ NPs modified SPCE & $0.004 \sim 40$ & $5.6 \times 10^{-3}$ & [41] \\
\hline Au-ATP-rGO composite modified Au Electrode & $0.1 \sim 200$ & 0.03 & [28] \\
\hline Self-supported np-Au microelectrode & $0.01 \sim 5$ & $5 \times 10^{-3}$ & [42] \\
\hline Layer-by-layer self-assembly modified Au electrode & $0.1 \sim 10.0$ & 0.03 & [43] \\
\hline $\begin{array}{c}\text { disposable screenprinted } \\
\text { Au electrodes }\end{array}$ & $0.001 \sim 100$ & $7 \times 10^{-4}$ & [44] \\
\hline 3D-rGO/AuNPs modified GCE & $0.001 \sim 10$ & $3.4 \times 10^{-4}$ & This work \\
\hline
\end{tabular}

\subsection{Selectivity and Reproducibility of the Electrochemical Aptasensors}

Selectivity and reproducibility are necessary factors to evaluate the efficiency of biosensors. Therefore, interferents OTB, DON, and ZEA were firstly employed to verify the selectivity of the designed aptasensor. The concentration for all the above interferents was set as $10 \mathrm{ng} / \mathrm{mL}$ while that for OTA was $1 \mathrm{ng} / \mathrm{mL}$. As seen from Figure 7, the responses of DON and ZEA were almost the same as that of control test. Unfavorably, the proposed assay has a small cross-reactivity with OTB as also observed in other publications [45,46], which might be ascribed to their similar molecular structures and common antigen epitope. However, considering the response signal of OTA is $~ 4.5$ fold of OTB with a 1/10 concentration, the selectivity of our system was acceptable. To check the reproducibility of the fabricated aptasensor, five replicates were performed under the same circumstances with responses of $160.15,157.08,159.59,160.99$ and $163.23 \Omega$, respectively. The relative standard deviation (RSD) was $1.393 \%$ for five measurements, indicating the high reproducibility of our sensor.

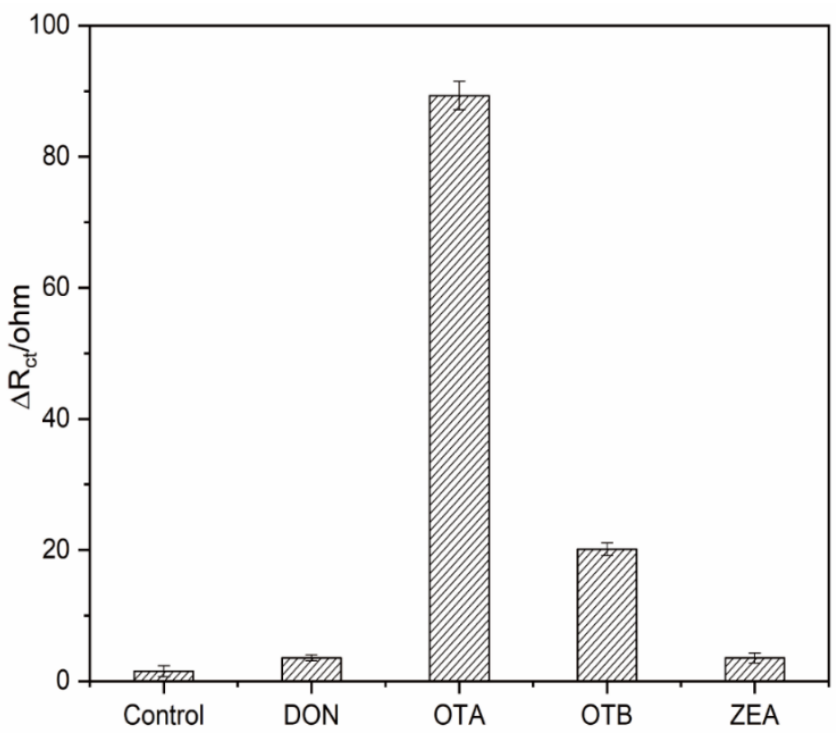

Figure 7. The specificity of the aptasensing system against OTA, ochratoxin B (OTB), deoxynivalenol $(\mathrm{DON})$, and zearalenone (ZEA).

\subsection{Real Sample Analysis}

The practicability and reliability of the impedimetric aptasensor was demonstrated in real red wine. After a simple filtration process was performed to remove the large molecules using a $0.22 \mu \mathrm{m}$ membrane, the filtered red wine samples were spiked with five different concentrations of OTA $(0.05,0.1,0.5,1$, and $2 \mathrm{ng} / \mathrm{mL})$. According to the above calibration curve, $0.05 \mathrm{ng} / \mathrm{mL}\left(\Delta \mathrm{R}_{\mathrm{CT}}=14.1 \pm 0.96 \Omega\right)$ was calculated as a much lower concentration of $0.001 \mathrm{ng} / \mathrm{mL}$, which demonstrated the above calibration curve is not applicable to the red wine samples. It is assumed that the matrix effect from red wine decreased the response 
sensitivity of the aptasensor [47]. Thus, the new calibration curve for red wine (Figure S4) is established for better quantification. The linear correlation equation can be indicated as $\Delta \mathrm{R}_{\mathrm{CT}}=39.09(\mathrm{x})+64.89 \log \left(\mathrm{c} / \mathrm{ng} \cdot \mathrm{mL}^{-1}\right)\left(\mathrm{R}^{2}=0.998\right)$ with a LOD of $0.023 \mathrm{ng} / \mathrm{mL}$, which was much lower than the European commission standard $(2 \mathrm{ng} / \mathrm{mL})$. The obtained recovery within the range of $93.14-112.75 \%$ was reasonable according to the investigation results given in Table 2, demonstrating good accuracy of developed aptasensor for OTA detection in real samples.

Table 2. Recoveries of OTA in red wine samples by the proposed aptasensor.

\begin{tabular}{cccc}
\hline Sample & Spike $(\mathbf{n g} / \mathbf{m L})$ & Determined $(\mathbf{n g} / \mathbf{m L})$ & Recovery $(\mathbf{\%})$ \\
\hline 1 & 0.05 & $0.050 \pm 0.002$ & 100.4 \\
2 & 0.1 & $0.099 \pm 0.013$ & 98.81 \\
3 & 0.5 & $0.53 \pm 0.053$ & 105.55 \\
4 & 1 & $0.93 \pm 0.072$ & 93.14 \\
5 & 2 & $2.26 \pm 0.253$ & 112.75 \\
\hline
\end{tabular}

\section{Conclusions}

In summary, this study developed a novel 3D-rGO/AuNPs-based aptasensing device using easy modification steps on the bare GCE to obtain sensitive and selective determination of OTA. The large-size of AuNPs embedded 3D network structures of porous graphene aerogel can afford large surface (active) area, good electrical conductivity, and tremendous binding sites for aptamer immobilization. With the presence of OTA, EIS signals of the proposed aptasensor were increased due to the obstruction of electron transfer caused by the specific binding between aptamer and OTA. Under optimal conditions, the developed aptasensing showed excellent EC performance with a high linear response and broad range from 0.001 to $10 \mathrm{ng} / \mathrm{mL}$ with an ultrasensitive LOD of $0.34 \mathrm{pg} / \mathrm{mL}$. The sensitive OTA aptasensor introduced in this work displayed rapidity, low cost, easy preparation, and good specificity with satisfied reproducibility, which could be potentially employed for detection of OTA in real samples as a portable and reliable tool. To be noted, the interference of OTB should be eliminated or reduced by developing on-column specific recognition of OTA with aptamer-based hybrid affinity monolithic column coupled with HPLC or LC for better application [48-50].

Supplementary Materials: The following are available online at https://www.mdpi.com/2079 $-6374 / 11 / 3 / 87 / s 1$, Table S1: Investigations of glucose role in thermal reduction process for 3D$\mathrm{rGO} / \mathrm{AuNPs}$ nanocomposites, Table S2: Optimized parameters of different volumes of $\mathrm{HAuCl}_{4} \cdot 4 \mathrm{H}_{2} \mathrm{O}$ for 3D-rGO/AuNPs nanocomposites, Table S3: Optimized parameters of different amounts of glucose for 3D-rGO/AuNPs nanocomposites; Figure S1: The optical images of sample a GO, b $\mathrm{GO}$ reduction without high temperature, $\mathrm{crGO} / \mathrm{AuNPs}$ with glucose and $\mathrm{d} \mathrm{rGO} / \mathrm{AuNPs}$ without glucose (detailed parameters were in Table S1), Figure S2: (A-C) The Nyquist plots of GCE after every step of fabrication of nanomaterial, aptamer, BSA, and OTA; (D) CV curves of sample b, c, and d fabricated GCE, Figure S3: Optimization of (A) concentrations of 3D-rGO/AuNPs, (B) concentrations of aptamer, (C) incubation time of aptamer, (D) incubation time of OTA with aptamer, Figure S4 Calibration curve of OTA detection in red wine based on $\Delta R_{C T}$ vs. $\log$ (OTA concentration).

Author Contributions: Conceptualization, Y.A. and D.Y.; methodology, S.Z.; writing-original draft preparation, Y.A. and Y.L.; writing - review and editing, R.A.-W., Y.G. and H.Z.; supervision, S.Z. and D.Y.; funding acquisition, S.Z. and D.Y. All authors have read and agreed to the published version of the manuscript.

Funding: This work is funded by the National Natural Sciences Foundation of China, grant number: 82073514; Natural Science Foundation of Zhejiang Province, grant number: LY17H260003; the Fundamental Research Funds for the Provincial Universities of Zhejiang, grant number: SJLY2021009 and K.C. Wong Magna Fund in Ningbo University.

Institutional Review Board Statement: Not applicable. 
Informed Consent Statement: Not applicable.

Conflicts of Interest: The authors declare no conflict of interest.

\section{References}

1. Iqbal, S.Z.; Asi, M.R.; Hanif, U.; Zuber, M.; Jinap, S. The presence of aflatoxins and ochratoxin a in rice and rice products, and evaluation of dietary intake. Food Chem. 2016, 210, 135-140. [CrossRef]

2. Fernández-Baldo, M.A.; Bertolino, F.A.; Messina, G.A.; Sanz, M.I.; Raba, J. Modified magnetic nanoparticles in an electrochemical method for the ochratoxin A determination in Vitis vinifera red grapes tissues. Talanta 2010, 83, 651-657. [CrossRef]

3. Yang, J.; Gao, P.; Liu, Y.; Li, R.; Ma, H.; Du, B. Label-free photoelectrochemical immunosensor for sensitive detection of Ochratoxin, A. Biosens. Bioelectron. 2015, 64, 13-18. [CrossRef] [PubMed]

4. Zhang, Y.; Qi, X.; Zheng, J.; Luo, Y.; Zhao, C.; Hao, J.; Li, X.; Huang, K.; Xu, W. Lipid Rafts Disruption Increases Ochratoxin A Cytotoxicity to Hepatocytes. J. Biochem. Mol. Toxicol. 2015, 30, 71-79. [CrossRef]

5. Armutcu, C.; Uzun, L.; Denizli, A. Determination of Ochratoxin A traces in foodstuffs: Comparison of an automated on-line twodimensional high-performance liquid chromatography and off-line immunoaffinity-high-performance liquid chromatography system. J. Chromatogr. A 2018, 1569, 139-148. [CrossRef]

6. Ahn, S.; Lee, S.; Lee, J.; Kim, B. Accurate determination of ochratoxin A in Korean fermented soybean paste by isotope dilution-liquid chromatography tandem mass spectrometry. Food Chem. 2016, 190, 368-373. [CrossRef] [PubMed]

7. Wu, L.; Li, G.H.; Xu, X.; Zhu, L.; Huang, R.M.; Chen, X.Q. Application of nano-ELISA in food analysis: Recent advances and challenges. Trends Anal. Chem. 2019, 113, 140-156. [CrossRef]

8. Jiang, L.; Qian, J.; Yang, X.W.; Yan, Y.T.; Liu, Q.; Wang, K. Amplified impedimetric aptasensor based on gold nanoparticles covalently bound graphene sheet for the picomolar detection of ochratoxin A. Anal. Chim. Acta 2014, 806, 128-135. [CrossRef]

9. Chen, W.; Yan, C.; Cheng, L.; Yao, L.; Xue, F.; Xu, J.G. An ultrasensitive signal-on electrochemical aptasensor for ochratoxin A determination based on DNA controlled layer-by-layer assembly of dual gold nanoparticle conjugates. Biosens. Bioelectron. 2018, 117, 845-851. [CrossRef]

10. Rhouati, A.; Yang, C.; Hayat, A.; Marty, J.L. Aptamers: A promising tool for ochratoxin A detection in food analysis. Toxins 2013, 5, 1988-2008. [CrossRef]

11. Wei, M.; Zhang, W.Y. A novel impedimetric aptasensor based on AuNPs-carboxylic porous carbon for the ultrasensitive detection of ochratoxin A. RSC Adv. 2017, 7, 28655. [CrossRef]

12. Hayat, A.; Andreescu, S.; Marty, J.L. Design of PEG-aptamer two-piece macromolecules as convenient and integrated sensing platform: Application to the label free detection of small size molecules. Biosens. Bioelectron. 2013, 45, 168-173. [CrossRef]

13. Qiao, X.; Wang, Y.; Arsalan, M.; Yang, S.; Wang, Y.; Sheng, Q.; Yue, T. A Label-free aptasensor for the detection of ochratoxin a based on competitive molecule-level interactions. J. Electrochem. Soc. 2020, 167, 147518. [CrossRef]

14. de Almeida, P.R.; Murad, A.M.; Silva, L.P.; Rech, E.L.; Alves, E.S. Development of a Graphene-Based Biosensor for Detecting Recombinant Cyanovirin-N. Biosensors 2020, 10, 206. [CrossRef] [PubMed]

15. Wu, Y.P.; Zhu, J.H.; Huang, L. A review of three-dimensional graphene-based materials: Synthesis and applications to energy conversion/storage and environment. Carbon 2019, 143, 610-640. [CrossRef]

16. Qiu, H.J.; Guan, Y.X.; Luo, P.; Wang, Y. Recent advance in fabricating monolithic 3D porous graphene and their applications in biosensing and biofuel cells. Biosens. Bioelectron. 2017, 89, 85-95. [CrossRef] [PubMed]

17. Liu, Q.S.; Zhong, H.G.; Chen, M.; Zhao, C.; Liu, Y.; Xi, F.N.; Luo, T. Functional nanostructure-loaded three-dimensional graphene foam as a non-enzymatic electrochemical sensor for reagentless glucose detection. RSC Adv. 2020, 10, 33739-33746. [CrossRef]

18. Shi, L.; Wang, Y.; Ding, S.M.; Chu, Z.Y.; Yin, Y.; Jiang, D.F.; Luo, J.Y.; Jin, W.Q. A facile and green strategy for preparing newly-designed 3D graphene/gold film and its application in highly efficient electrochemical mercury assay. Biosens. Bioelectron. 2017, 89, 871-879. [CrossRef]

19. You, M.; Yang, S.; Tang, W.X.; Zhang, F.; He, P.G. Molecularly imprinted polymers-based electrochemical DNA biosensor for the determination of BRCA-1 amplified by $\mathrm{SiO}_{2} @$ Ag. Biosens. Bioelectron. 2018, 112, 72-78. [CrossRef]

20. Perumal, V.; Saheed, M.S.M.; Mohamed, N.M.; Saheed, M.S.M.; Murthe, S.S.; Gopinath, S.C.B.; Chiu, J.M. Gold nanorod embedded novel 3D graphene nanocomposite for selective bio-capture in rapid detection of Mycobacterium tuberculosis. Biosens. Bioelectron. 2018, 116, 116-122. [CrossRef]

21. Yue, H.Y.; Zhang, H.; Huang, S.; Lin, X.Y.; Gao, X.; Chang, J.; Yao, L.H.; Guo, E.J. Synthesis of ZnO nanowire arrays/3D graphene foam and application for determination of levodopa in the presence of uric acid. Biosens. Bioelectron. 2017, 89, 592-597. [CrossRef]

22. Ensafi, A.A.; Akbarian, F.; Heydari-Soureshjani, E.; Rezaei, B. A novel aptasensor based on 3D-reduced graphene oxide modified gold nanoparticles for determination of arsenite. Biosens. Bioelectron. 2018, 122, 25-31. [CrossRef] [PubMed]

23. Karczmarczyk, A.; Reiner-Rozman, C.; Hageneder, S.; Dubiak-Szepietowska, M.; Dostalek, J.; Feller, K.H. Fast and sensitive detection of ochratoxin A in red wine by nanoparticle-enhanced SPR. Anal. Chim. Acta 2016, 937, 143-150. [CrossRef]

24. Al-Ani, L.A.; AlSaadi, M.A.; Kadir, F.A.; Hashim, N.M.; Julkapli, N.M.; Yehye, W.A. Graphene- gold based nanocomposites applications in cancer diseases; Efficient detection and therapeutic tools. Eur. J. Med. Chem. 2017, 139, 349-366. [CrossRef] [PubMed]

25. Kasturi, S.; Eom, Y.; Torati, S.R.; Kim, C.G. Highly sensitive electrochemical biosensor based on naturally reduced rGO/Au nanocomposite for the detection of miRNA-122 biomarker. J. Ind. Eng. Chem. 2021, 93, 186-195. [CrossRef]

26. Huang, X.; Qi, X.; Boey, F.; Zhang, H. Graphene-Based Composites. Chem. Soc. Rev. 2012, 41, 666-686. [CrossRef] 
27. Guo, W.; Wu, L.; Fan, K.; Nie, D.; He, W.; Yang, J.; Zhao, Z.; Han, Z. Reduced graphene oxide-gold nanoparticle nanoframework as a highly selective separation material for aflatoxins. Sci. Rep. 2017, 7, 14484. [CrossRef] [PubMed]

28. Qian, J.; Jiang, L.; Yang, X.W.; Yan, Y.T.; Mao, H.P.; Wang, K. Highly sensitive impedimetric aptasensor based on covalent binding of gold nanoparticles on reduced graphene oxide with good dispersity and high density. Analyst 2014, 139, 5587-5593. [CrossRef]

29. Wu, X.; Xing, Y.; Pierce, D.; Zhao, J.X. One-Pot Synthesis of Reduced Graphene Oxide/Metal (Oxide) Composites. ACS Appl. Mater. Interfaces 2017, 9, 37962-37971. [CrossRef]

30. Zhang, B.; Wang, X.; Liu, F.; Cheng, Y.; Shi, A.D. Effective reduction of nonspecific binding by surface engineering of quantum dots with bovine serum albumin for cell-targeted imaging. Langmuir 2012, 28, 16605-16613. [CrossRef]

31. Lichtenberg, J.Y.; Ling, Y.; Kim, S. Non-Specific Adsorption Reduction Methods in Biosensing. Sensors 2019, 19, 2488. [CrossRef] [PubMed]

32. Tang, W.; Peng, L.; Yuan, C.; Wang, J.; Mo, S.; Zhao, C. Facile synthesis of 3d reduced graphene oxide and its polyaniline composite for super capacitor application. Synth. Met. 2015, 202, 140-146. [CrossRef]

33. Shen, J.F.; Yan, B.; Shi, M.; Ma, H.W.; Li, N.; Ye, M.X. One step hydrothermal synthesis of $\mathrm{TiO}_{2}$-reduced graphene oxide sheets. J. Mater. Chem. 2011, 21, 3415-3421. [CrossRef]

34. Yang, S.; Yue, W.; Huang, D.; Chen, C.; Lin, H.; Yang, X. A facile green strategy for rapid reduction of graphene oxide by metallic zinc. RSC Adv. 2012, 2, 8827-8832. [CrossRef]

35. Sahoo, P.K.; Aepuru, R.; Panda, H.S.; Bahadur, D. Ice-templated synthesis of multifunctional three-dimensional graphene/noble metal nanocomposites and their mechanical, electrical, catalytic, and electromagnetic shielding properties. Sci. Rep. 2015, 5, 17726. [CrossRef] [PubMed]

36. Ge, X.L.; Li, Z.Q.; Yin, L.W. Metal-organic frameworks derived porous core/shellCoP@C polyhedrons anchored on 3D reduced graphene oxide networks as anode for sodium- ion battery. Nano Energy 2017, 32, 117-124. [CrossRef]

37. Guo, D.M.; Cai, P.J.; Sun, J.; He, W.N.; Wu, X.H.; Zhang, T.; Wang, X.; Zhang, X.T. Reduced-graphene-oxide/metal-oxide p-n heterojunction aerogels as efficient 3D sensing frameworks for phenol detection. Carbon 2016, 99, 571-578. [CrossRef]

38. Vilian, A.T.E.; An, S.; Choe, S.R.; Kwak, C.H.; Huh, Y.S.; Lee, J.; Han, Y.K. Fabrication of 3D honeycomb-like porous polyurethanefunctionalized reduced graphene oxide for detection of dopamine. Biosens. Bioelectron. 2016, 86, 122-128. [CrossRef] [PubMed]

39. Rashid, J.I.A.; Vayithiswary, K.; Muhammad, H.A.; Aye, A.M.; Safura, T.; Azizi, M.; Keat, K.O.; Nor Azah, Y. An electrochemical sensor based on gold nanoparticles-functionalized reduced graphene oxide screen printed electrode for the detection of pyocyanin biomarker in Pseudomonas aeruginosa infection. Mat. Sci. Eng. C 2021, 120, 111625. [CrossRef]

40. Zamfir, L.G.; Puiu, M.; Bala, C. Advances in Electrochemical Impedance Spectroscopy Detection of Endocrine Disruptors. Sensors 2020, 20, 6443. [CrossRef]

41. Rivas, L.; Mayorga-Martinez, C.C.; Quesada-González, D.; Zamora-Gálvez, A.; de la Escosura-Muñiz, A.; Merkoçi, A. Labelfree impedimetric aptasensor for ochratoxin-A detection using iridium oxide nanoparticles. Anal. Chem. 2015, 87, 5167-5172. [CrossRef] [PubMed]

42. Shi, L.; Rong, X.J.; Wang, Y.; Ding, S.M.; Tang, W.Y. High-performance and versatile electrochemical aptasensor based on self-supported nanoporous gold microelectrode and enzyme-induced signal amplification. Biosens. Bioelectron. 2018, 102, 41-48. [CrossRef] [PubMed]

43. Suea-Ngam, A.; Howes, P.D.; Stanley, C.E.; de Mello, A.J. An exonuclease I-assisted silver-metallized electrochemical aptasensor for ochratoxin a detection. ACS Sens. 2019, 4, 1560-1568. [CrossRef]

44. Nan, M.; Bi, Y.; Xue, H.L.; Xue, S.L.; Long, H.T.; Pu, L.M.; Fu, G. Rapid determination of ochratoxin A in grape and its commodities based on a label-free impedimetric aptasensor constructed by layer-by-layer self-assembly. Toxins 2019, 11, 71. [CrossRef]

45. Tozlovanu, M.; Pfohl-Leszkowicz, A. Ochratoxin A in roasted coffee from French supermarkets and transfer in coffee beverages: Comparison of analysis methods. Toxins 2010, 2, 1928-1942. [CrossRef] [PubMed]

46. Sun, A.L.; Zhang, Y.F.; Sun, G.P.; Wang, X.N.; Tang, D.P. Homogeneous electrochemical detection of ochratoxin A in foodstuff using aptamer-graphene oxide nanosheets and DNaseI-based target recycling reaction. Biosens. Bioelectron. 2018, 89, 659-665. [CrossRef] [PubMed]

47. Alhamoud, Y.; Yang, D.; Kenston, S.S.F.; Liu, G.; Liu, L.; Zhou, H.; Ahmed, F.; Zhao, J. Advances in biosensors for the detection of ochratoxin A: Bio-receptors, nanomaterials, and their applications. Biosens. Bioelectron. 2019, 141, 111418. [CrossRef] [PubMed]

48. Lyu, H.; Sun, H.; Zhu, Y.; Wang, J.; Xie, Z.; Li, J. A double-recognized aptamer-molecularly imprinted monolithic column for high-specificity recognition of ochratoxin A. Anal. Chim. Acta 2020, 1103, 97-105. [CrossRef]

49. Chi, J.; Chen, M.; Deng, L.; Lin, X.; Xie, Z. A facile AuNPs@ aptamer-modified mercaptosiloxane-based hybrid affinity monolith with an unusually high coverage density of aptamer for on-column selective extraction of ochratoxin A. Analyst 2018, 143, 5210-5217. [CrossRef]

50. Chen, Y.; Ding, X.; Zhu, D.; Lin, X.; Xie, Z. Preparation and evaluation of highly hydrophilic aptamer-based hybrid affinity monolith for on-column specific discrimination of ochratoxin A. Talanta 2019, 200, 193-202. [CrossRef] 